\title{
La capitalización del conocimiento en la empresa de medios. Guía para su rentabilización estratégica
}

\section{The capitalization of mass media knowledge. A guide to strategic profitability}

\author{
Dra. Miriam Rodríguez Pallares \\ Universidad Internacional de la Rioja \\ miriam.rodriguez@unir.net \\ ORCID: https://orcid.org/0000-0002-5486-0298 \\ Dra. María José Pérez Serrano \\ Universidad Complutense de Madrid \\ mariajoseperezserrano@pdi.ucm.es \\ ORCID: https://orcid.org/0000-0002-2190-7619
}

Resumen: La gestión de los activos y recursos intangibles en el entorno empresarial es ya un paradigma de común aceptación en el ámbito académico y profesional. La marca, el conocimiento o el capital relacional son elementos que departamentos financieros y gerentes contemplan en sus estrategias. En este contexto, se focaliza la atención en la gestión del conocimiento en la empresa informativa. El protagonismo del saber organizacional como base de la economía encuentra sus orígenes en la época post-industrial y, con el tiempo, la denominada "Sociedad del Conocimiento" ha llegado a consolidar su significado aludiendo a los cambios sociales y económicos suscitados por la popularización de las TIC, a los procesos de creación de conocimiento como factor productivo, la gestión de este factor en un entorno organizacional, y al trabajo basado en él. Por lo tanto, se entiende que la impronta de la capitalización del conocimiento no puede ser ajena a la industria mediática. A partir de la revisión bibliográfica, se propone una guía de buenas prácticas para implementar un modelo de gestión del conocimiento eficaz en la empresa informativa innovadora (adjetivada de forma metonímica como "del siglo XXI"). Se contemplan para esta propuesta las particularidades identitarias de la industria mediática y la relevancia que el factor tecnológico, la cultura corporativa y el liderazgo -como acicate del sentimiento de pertenencia- suponen por cimientos para la correcta aplicación de un modelo de gestión coordinado, sistemático y transversal. 
Abstract: The management of intangible assets and resources in a business environment is clearly recognized an academic and professional context. Financial and general managers are nowadays paying attention to concepts like brand, knowledge or relational capital to design their business strategies. In this context, the main focus of this article is on knowledge management as applied to a media company. The importance of knowledge as the basis of economy can be located in the post-industrial area and, overtime, the so-called "Knowledge Society" has consolidated its meaning that now is referring to social and economic changes as a result of the popularization of the TICs, the new knowledge creation process as a productive factor, the knowledge management in business environment and the knowledge based work. Therefore, it is understood that the capitalization of the knowledge should be taken into account in the media industry too. Based on a literature review, we propose a guide to good practice for the implementation of an effective knowledge management model in innovative media companies. In preparing this proposal we have considered the particular characteristics of the media industry and the relevance of the technological factor, corporative culture and leadership in the correct application of a coordinated, systematic and transversal knowledge management model.

Palabras clave: Gestión del Conocimiento, Empresa Informativa, Capital Intelectual.

Keywords: Knowledge Management, Media Company, Intellectual Capital.

\section{INTRODUCCIÓN Y ESTADO DE LA CUESTIÓN}

$\mathrm{Si}$ bien el origen del concepto "Sociedad del Conocimiento" se ubica en las teorías económicas de la época post-industrial (Drucker, 1969), cuando se postulaba un mayor protagonismo de la economía de servicios y donde la alta cualificación y la especialización teórica superaban en importancia al trabajo físico, con el tiempo, este significante ha llegado a dilatar su significado hasta hacer referencia a los cambios sociales y económicos suscitados por la popularización de las TIC -aspecto que justifica fundamentalmente la aplicación del término "Sociedad de la Información"-; a los procesos de creación de conocimiento como factor productivo; a la gestión de este factor en un entorno organizacional, y al trabajo basado en él (Heidenreich en Krüger, 2006).

\subsection{El valor del conocimiento en el entorno organizacional}

Aceptado, por tanto, el valor del conocimiento como activo corporativo y garante de ventaja diferencial, su capitalización es un hecho. Lo que significa que es posible codificarlo en términos económicos. Es, por ello, que la gestión del conocimiento en un entorno de producción se convierte en condicionante de su capacidad de innovación (Carrillo-Durán y Nuño Moral, 2010; Sancho, 2007) y adaptación a un entorno en el que las organizaciones han dejado de ser agentes económicos, para asumir también un papel relevante en el ámbito sociopolítico e, incluso, académico al responder a las peticiones de una sociedad cada vez más exigente.

El origen de la capitalización del conocimiento (Gorz, 2001 en Krugër, 2006), o de la economía basada en el conocimiento, se puede relacionar con la Teoría de 
los Recursos y Capacidades o con un más reciente enfoque basado en el valor de los activos intangibles, entre ellos, el capital intelectual o el todo, y el conocimiento o la parte (Navas y Ortiz, 2002; Camelo, 1996), fundamentado en la teoría administrativa de la learning organization (Senge, 2005). Sin entrar en esta disquisición -es posible retrotraerse incluso a la filosofía griega y el estudio de la gnosis-, la gestión del conocimiento es un proceso estratégico integral cuyo fin último es el de "dar valor al saber hacer de una organización y de salvaguardarlo, explicitándolo o democratizando el no explicitable, a través de buenas prácticas colaborativas" (Rodríguez, 2014).

La gestión del conocimiento puede considerarse, así, una pieza imprescindible en el concepto estratégico de la actividad empresarial, entendida esta como "el conjunto de acciones ofensivas o defensivas de la empresa para posicionarse y/o anticiparse al mercado con el fin de crear y desarrollar una ventaja competitiva a largo plazo" (Porter, 1999) que exige, inevitablemente, de la innovación como elemento de diferenciación para sobrevivir en el mercado.

En este sentido, la gestión del conocimiento está íntimamente relacionada con la cultura y los valores de una organización (Nonaka y Takeuchi, 1999) y con el liderazgo transversal, es decir, aquel que se "extiende más allá de todo límite jerárquico o funcional" (Gauchi, 2012). Estos elementos son condicionantes en el proceso de identificación, gestión y rentabilización del conocimiento.

Sveiby (1997) identificaba, como parte del Capital Intelectual, y en relación al origen del mismo, tres tipos de conocimiento: el conocimiento humano también capital humano-, propio de las habilidades y competencias de los trabajadores, esto es, su talento; el conocimiento estructural -también capital estructural-, el identificado con la actividad interna de la organización, es decir, con su capacidad organizativa y estratégica, y el conocimieno relacional -también capital relacional- o aquel originado a partir de las relaciones de la organización con sus públicos de interés externos y que es una importante herramienta en la constitución de su reputación. Por lo tanto, y de acuerdo a la teoría de los stakeholders (Freeman, 1984), la gestión del conocimiento y su democratización o transparencia debe ser considerada una actividad que se nutra y repercuta en el público de interés interno y externo de la corporación.

\begin{tabular}{|c|c|c|c|c|}
\hline $\begin{array}{l}\text { Propiedades } \\
\text { intrínsecas }\end{array}$ & Origen & $\begin{array}{c}\text { Dimensión } \\
\text { ontológica }\end{array}$ & $\begin{array}{l}\text { Aportación en } \\
\text { la cadena de } \\
\text { producción }\end{array}$ & Contenido \\
\hline $\begin{array}{l}\text { Explícito/ } \\
\text { información } \\
\text { Tácito/capital } \\
\text { humano }\end{array}$ & $\begin{array}{l}\text { Interno } \\
\text { Externo }\end{array}$ & $\begin{array}{l}\text { Individual } \\
\text { Colectivo }\end{array}$ & $\begin{array}{l}\text { Generativo } \\
\text { Productivo } \\
\text { Representativo }\end{array}$ & $\begin{array}{l}\text { Conceptual- } \\
\text { metodológico } \\
\text { Operacional } \\
\text { Instrumental } \\
\text { (captado o } \\
\text { creado) }\end{array}$ \\
\hline $\begin{array}{l}\text { Nonaka y Takeuchi, } \\
1999 \\
\text { Pérez-Montoro, } \\
2006\end{array}$ & $\begin{array}{l}\text { Andreu y } \\
\text { Sieber, } 1999\end{array}$ & $\begin{array}{l}\text { Spender y } \\
\text { Grant, } 1996\end{array}$ & $\begin{array}{l}\text { Wikstrom y } \\
\text { Norman, } 1994\end{array}$ & $\begin{array}{l}\text { Núñez Paula, } \\
2004\end{array}$ \\
\hline
\end{tabular}

Tabla 1. Selección de clasficaciones del conocimiento. Fuentes: Nonaka y Takeuchi, 1999; Pérez-Montoro, 2006; Andreu y Sieber, 1999; Spender y Grant, 1996; Wikstrom y Normam, 1994 y Núñez Paula, 2004. 
A la hora de establecer etapas en la gestión del conocimiento (su know-how) se encuentran diferentes propuestas en la literatura académica que redundan en la identificación; adquisición (creación o compra); codificación; almacenamiento o archivo; recuperación; distribución o transferencia; aplicación, y control (Nonaka y Takeuchi, 1999; Davenport y Prusak, 2001; Barceló-Llauger, 2001; Rodríguez, 2006; Barragán, 2009). Lo que Rusly, Corner y Sun (2012) resumieron en las tres siguientes fases: adquisición, creación e intercambio de conocimientos.

En relación a la transformación de conocimiento, Nonaka y Takeuchi (1999) propusieron el modelo que ha servido de base conceptual a teorías posteriores. Hablaban así de cuatro procesos: la socialización (tácito a tácito); la externalización (tácito a explícito); la internalización (explítico a tácitco), y la combinación (explícito a explícito). Aunque fueron muchas las teorías que siguieron a esta propuesta, la clasificación de los autores asiáticos sigue significando la base teórica sobre la que orbitan otras propuestas, como la de Choo (1999), que incluye en su modelo la toma de decisiones a partir del conocimiento adquirido, complementando así el proceso de su rentabilización. En lo que respecta a la funcionalidad de la gestión del conocimiento, Tissen, Andriesssen y Dreppez (en Mousavizadeh, Harden, Ryan y Windsor, 2015) propusieron una división que nos sirve para justificar la propuesta teórica que se incluye en estas páginas. Los autores hablaban de una gestión operativa del conocimiento, por un lado, y de una gestión estratégica del conocimiento, por otro. Mientras lagestión operativa focaliza sus objetivos en identificar, conservar $y$ transferir el conocimiento humano de la organización a fin de optimizar la eficacia -entendida como el grado en que el output satisface las necesidades del clientey la eficiencia -utilizar los factores de producción en combinaciones de menor coste (Todaro, 1997)- en la producción; la gestión estratégica busca explotar el conocimiento en beneficio de la estrategia de negocio, esto es, va más allá del proceso productivo y se centra en la búsqueda de las ventajas competitivas que permitan a la organización posicionarse de un modo confortable en el mercado.

\subsection{La gestión del conocimiento en el entorno de los media}

Para contextualizar esta propuesta teórica parece pertinente dejar claras dos características identitarias de las empresas de información:

Característica 1_Dualidad de fines. Por un lado, como unidad de producción inserta en la economía de mercado, los media y sus empresas cuentan con una estructura propia y una batería de elementos que oscilan con el paso del tiempo y que se reflejan en sus modelos de dirección y gestión (Albarran, 2005; Nieto e Iglesias, 2000; Picard, 2002; Pringle y Starr, 2004; Sánchez-Tabernero, 2000; Sylvie et al., 2008) cuyo objetivo es, en última instancia, obtener un beneficio económico que les permita alcanzar un posicionamiento estable en el mercado y maximizar sus ventajas competitivas. Por otro lado, las empresas de información se plantean como misión producir contenidos noticiosos, formativos o de entretenimiento que contribuyen a completar uno de los derechos fundamentales del ser humano: recibir información veraz y plural (Pérez Serrano, 2015), este hecho justifica la impronta de su actividad en la construcción de las bases sociales. 
Característica 2_La producción de los media es intangible por naturaleza, esto justifica que la gestión de contenidos o documentación corporativa tienda a focalizar sus actividades en los contenidos mediáticos o mediatizables, es decir, en su producción, obviando en un modelo sistemático y centralizado los contenidos generados a partir de procesos productivos o de explicitación del conocimiento provenientes de otras áreas de actividad de la organización.

Correlacionar estas características con la clasificación de la gestión del conocimiento propuesta por Tissen, Andriesssen y Dreppez (en Mousavizadeh, Harden, Ryan y Windsor, 2015) nos permite identificar un punto de partida: la gestión del conocimiento en la empresa informativa debe contemplar todas las dimensiones necesarias para responder a la actividad funcional y a la estratégica y este proceso, además, debe contemplar el traslado de resultados de forma voluntaria a los públicos de interés, interesados en obtener información relativa a los recursos que crean riqueza en las empresas como parte del proceso de rendición de cuentas (Tejedo Romero, 2014) que les legitima para operar en el mercado.

Se trata, en definitiva, de sobrepasar el límite de la gestión de contenidos mediáticos o mediatizables e ir un paso más allá para incluir en el proceso de gestión y control al conocimiento explícito generado en otras áreas departamentales y democratizar el tácito.

Esta lógica gradual responde al debate abierto en relación a quién depende de quién en lo relativo a la gestión de contenidos o documentación y a la gestión del conocimiento. Sin pretender abordar este debate, en estas líneas se reconoce que la gestión de contenidos es previa a la gestión del conocimiento en términos de teorización científica y su coadyuvante indiscutible en términos prácticos (Rodríguez Pallares, 2014), ya que las técnicas documentales y la explotación de las TIC son imprescindibles en el proceso de codificación, custodia y transferencia del conocimiento explícito (Falconer, 2000; Davenport y Prusak, 2001; Rodríguez, 2006).

Se asume, entonces, una relación de dependencia o complementariedad y se entiende que la gestión del conocimiento está en un plano superior, dado que contempla un fin más ambicioso: la identificación, la redirección, la reutilización y la integración del conocimiento tácito en los procesos de negocio (Douglas, 2002).

No existe un modelo estándar para democratizar el saber individual, pero no cabe duda de que, para evitar la perennidad de la línea filosófica de que "uno vale para la empresa lo que sabe y no saben los demás" (Rodríguez Pallares, 2014), se hace necesario cuidar la cultura y el clima organizacional, esto es, fomentar el engagement del trabajador.

En el sector de los media, donde el número de contratados autónomos y falsos autónomos se acrecentó de un $25,6 \%$ a un $26,8 \%$ en 2016 (APM, 2016), donde las consecuencias del apalancamiento financiero se traducen en despidos, y donde un segundo problema reconocido por el sector profesional se focaliza en la mala retribución del trabajo periodístico (APM, 2016), parece que la estabilidad necesaria para fomentar un clima conveniente para la transmisión de conocimiento todavía debe seguir trabajándose.

\section{OBJETIVOS Y METODOLOGÍA}

En esta ocasión, se plantea un trabajo eminentemente teórico, que no fija proposiciones conjeturales (Kerlinger y Lee, 2005) como punto de partida, sino 
que trata de responder a las necesidades de los modelos de gestión del conocimiento que se podrían implementar en los medios de comunicación a modo de propuesta, hoja de ruta o guía de buenas prácticas. Todo ello fundamentado en la revisión bibliográfica.

Por lo tanto, no se abordan en ningún momento dimensiones de análisis propias de cada caso, que impliquen incorporar ad hoc estos modelos a sus estrategias de negocio. Esto es, quedan fuera del interés de esta propuesta el estudio de la estructura económica propia de la empresa de medios o los comportamientos estructurales del sector. Se trata, en definitiva, de aportar una aproximación teórica de lo que debe ser, es decir, de acercar la perspectiva teórico-académica a la aplicación práctica-profesional de los medios de comunicación.

\section{PROPUESTA CONCEPTUAL DE UN MODELO DE GESTIÓN DEL CONOCIMIENTO EN LOS MEDIA}

Todo proceso organizacional es una actividad coordinada, que involucra a personas y recursos tecnológicos. Dado que la gestión del conocimiento se considera una actividad estratégica, debe partir de la alta dirección y expandirse de forma transversal por todas las áreas de actividad organizacional, esto es, no debe cincunscribirse a un área departamental concreta, puesto que se alimenta y comparte sus resultados con toda la organización.

Los objetivos de la gestión del conocimiento en el sector de los media se pueden resumir en los siguientes:

- Medir y gestionar el conocimiento interno.

- Generar y atraer conocimiento externo.

- Explotar el conocimiento organizacional para fomentar la innovación operativa y estratégica.

\begin{tabular}{|c|c|c|}
\hline ORIGEN & NATURALEZA & OBJETO Y PROCEDIMIENTO \\
\hline \multirow[t]{2}{*}{ Interno } & Explícito & $\begin{array}{l}\text { Establecer modelos de codificación, descripción y } \\
\text { archivo del conocimiento albergado en el repositorio } \\
\text { central facilitando su reutilización. }\end{array}$ \\
\hline & Tácito & $\begin{array}{l}\text { Técnicas de socialización y democratización del } \\
\text { conocimiento a través de relaciones interpersonales } \\
\text { (directas, digitales o en diferido). Bien sea de forma } \\
\text { interdepartamental, bien sea entre los diferentes } \\
\text { puntos de la red de emisoras radiofónicas de la } \\
\text { misma marca o grupo radiofónico. }\end{array}$ \\
\hline \multirow[t]{2}{*}{ Externo } & Pasivo / Explícito & $\begin{array}{l}\text { Captación a través de prácticas de vigilancia } \\
\text { competitiva. }\end{array}$ \\
\hline & Activo / Tácito & $\begin{array}{l}\text { Captación mediante ejercicios de formación (cursos, } \\
\text { conferencias, congresos, grupos de debate...). }\end{array}$ \\
\hline
\end{tabular}

Tabla 2. Clasificación, objeto y procedimiento de la gestión del conocimiento en el entorno mediático. Fuentes: Rodríguez Pallares, 2016.

Como todo proceso de gestión a largo plazo, Fase1_Diagnóstico inicialoauditoríadel conocimiento. la implementación de un modelo de gestión del Barceló Llauger (2001) contemplaba tres herramientas conocimiento implica una planificación temporal que fundamentales para llevar a cabo esta etapa: mapas debe contemplar las siguientes fases: del conocimiento, matrices y cuestionarios. 
Fase 2_Selección, codificación y archivo del conocimiento explícito. Este proceso, como se mencionaba anteriormente, depende directamente de las prácticas profesionales de gestión documental y de las prestaciones tecnológicas. Es el punto clave que vincula la gestión de contenidos y la gestión del conocimiento, y abre el debate sobre su relación de complementareidad o subordinación. Nuevas corrientes teóricas apuestan por buscar sinergias entre distintos modelos de gestión de intangibles, así se llega a proponer la explotación de los BPMS (Business Process Management Systems) como soporte tecnológico para gestionar y difundir conocimiento a todos los estadios organizacionales (Martín, Lechuga y Medina, 2017).

Fase 3_Explicitación del conocimiento tácito susceptible de volcarse en un soporte, y socialización y creación del conocimiento tácito. La posibilidad de volcar en un soporte el conocimiento tácito es siempre una prioridad, porque asegura su permanencia en el haber de la organización, independientemente de la fluctuación de las personas en su plantilla. No obstante, explicitar el talento en su totalidad es imposible, por lo que se hacen necesarias acciones coordinadas de socialización. El ambiente laboral es, aquí, condicionante de la eficacia de este proceso que, a grandes rasgos, se podría comparar con una labor de mentoring sistematizada y controlada. Fase 4_Medición, control y explotación del conocimiento. Cualquier proceso que implique planificación, exige un diagnóstico previo y un control de las acciones realizadas en un tiempo determinado con el fin de evaluar la eficacia de las mismas. Por ello, se plantea la aplicación de auditorías periódicas que justifiquen el valor del conocimiento humano, estructural y relacional, un valor no cuantificable en términos de contabilidad financiera, pero sí en términos económicos -se capitaliza, tiene valor en el mercado, porque influye en el proceso de negocio de las organizaciones- dado que la empresa no puede garantizar su control.

En este sentido, López Triana y Sotillo (2009), de Villafañe \& Asociados, aclaran que:

"Desde la práctica de la gestión profesional de los intangibles empresariales podemos concretar éstos en cuatro: la marca, la gestión del conocimiento, la responsabilidad corporativa y la reputación corporativa. De los cuatro, sólo la marca puede ser considerado un activo intangible en la medida en que su valor económico, no todavía pero sí dentro de poco tiempo, podrá figurar y deberá figurar en el balance de cualquier empresa cotizada. Los otros tres -conocimiento, responsabilidad y reputaciónson recursos intangibles, y de la misma manera que ocurre con los activos intangibles, pueden alcanzar mucho valor; la diferencia es que los recursos intangibles no admiten una evaluación económico financiera $y$, en consecuencia, no figuran en el balance, pero deben ser igualmente gestionados porque de esa gestión depende, en gran medida, el valor que puedan llegar a alcanzar".

\section{Explícito Tácito}

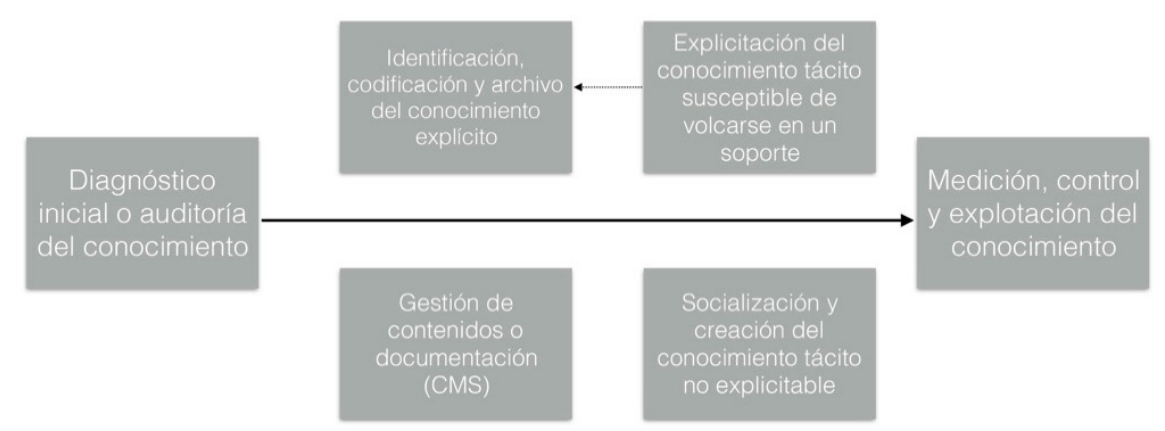

Gráfico1. Fases de la gestión del conocimiento de acuerdo a la naturaleza del mismo. Fuente: elaboración propia. 
De acuerdo a las líneas maestras planteadas anteriormente, la siguiente propuesta conceptual se estructura en función de la naturaleza del conocimiento: por un lado, la gestión del conocimiento explícito y, por el otro, la gestión del conocimiento tácito.

\subsection{La gestión del conocimiento explícito}

La gestión del conocimiento explícito entronca con la gestión de contenidos o documentación. En esta ocasión, se propone la sistematización en el proceso de descripción y archivo de contenidos a través de "un metadataje adecuado, un formato estandarizado y un modelo de almacenamiento y accesibilidad normalizado" (García Lastra, 2012). Se trata de fomentar la centralización de contenidos originados en todas las áreas de actividad organizacional. Según Rodríguez Pallares (2014), el conocimiento se compone de:

- Datos: significantes con unidad mínima de significado.

- Información: contextualización de los datos, con el fin concreto de alcanzar entendimiento. Desde el punto de vista del receptor, la información pretende cambiar la perspectiva del destinatario tras una asimilación individual. $Y$, desde el punto de vista del emisor, la información es la representación contextualizada y dotada de significado de un conjunto de datos, de una idea o conjunto de ideas.

- Conocimiento explicitado: interiorización de la información, razón de la sabiduría y que, aplicada a un contexto o a una actividad concreta, se convierte en talento.

Parece lógico pensar que la usabilidad de los contenidos variará considerablemente en función de su procedencia y naturaleza, por lo que se plantea como fundamental que la trazabilidad de los mismos esté claramente definida y se creen perfiles de acceso que veten, bien su acceso, bien su modificación, en función del perfil profesional solicitante.

Así pues, el componente tecnológico adquiere una relevancia incuestionable como repositorio del saber organizacional y resultado de su actividad, superando así las prestaciones de un MAM (Media Asset Management), dado que se contempla la inclusión de formatos ajenos a los contenidos mediáticos o mediatizables. La centralización de contenidos (incluyendo el conocimiento explícito) hace referencia a la sistematización en la descripción y almacenamiento, y exige de capacitación profesional con criterios operativos y estratégicos a fin de asegurar su selección, descripción, vinculación y recuperación de forma alineada a los intereses organizacionales.

De este modo, se evidencia que la gestión de contenidos no debe reducir su objeto de trabajo a contenidos mediáticos o mediatizables, sino que, apelando al fin de las empresas informativas de obtener un rédito económico por su actividad, deben gestionar todo el saber explicitado susceptible de reducir el riesgo en la toma de decisiones futuras (Rodríguez Pallares y Pérez Serrano, 2017). 


\subsection{La gestión del conocimiento tácito}

La gestión del saber individual es compleja y exige la confluencia de varios elementos organizacionales que diferentes autores han identificado como garantes de su efectividad: la cultura organizacional orientada al aprendizaje y la innovación; el liderazgo transversal; la estructura, o la tecnología (Andersen, 1995; Tejedor y Aguirre, 1998).

Para Robbins y Judge (2009), la cultura es "un sistema de significado compartido por los miembros, el cual distingue a una organización de las demás", y parte de la estimulación y motivación de los trabajadores -un grado óptimo de egangement- para alcanzar, en último término, una estabilidad organizacional que alinee la cultura con la perspectiva estratégica del sujeto de negocio.

En este sentido, es entonces imprescindible la definición de los valores organizacionales, el cumplimiento de los mismos en sentido ascendente y descendente, y la presencia de líderes con capacidad empática e inteligencia emocional (Goleman, Boyatzis y McKee, 2003; Jericó, 2000), que sean capaces de estimular y motivar a las personas a fin de hacerlas sentir parte de la organización, disfrutar de sus logros, y sufrir con sus fracasos, de tal modo que, además de por la vinculación contractual que les une con la organización, deseen compartir su talento por voluntad propia.

Quizás es aquí donde la inestabilidad del sector mediático, la tendencia a la externalización y la fluctuación de personas habitual en las productoras beneficiarias de ese outsourcing dificultan la estabilización de esta ambiente propicio que, no obstante, debe consolidarse como conditio sine qua non para la alcanzar ese proceso de socialización (tácito-tácito) planteado por Nonaka y Takeuchi (1999). Implementar un proyecto de estas características implica, por tanto, la aplicación de cambios en la cultura organizacional, la gestión de personas, y la adaptación a nuevos procesos de trabajo y prestaciones tecnológicas. Esto es: una nueva forma de hacer.

\section{DISCUSIÓN Y CONCLUSIONES}

La capitalización del conocimiento, es decir, su puesta en valor en el entorno organizacional, es una cuestión ampliamente aceptada en el ámbito académico y valorada en el ámbito empresarial. Sin embargo, en el contexto mediático, la gestión del saber organizacional no parece gozar de una alta tasa de implementación y consolidación como actividad estratégica e integral coordinada, sistematizada y transversal.

Lejos de proponer un modelo pormenorizado, se han tratado de plasmar las directrices que, a nuestro parecer y desde una perspectiva conceptual, teórica $y$, por lo tanto, genérica, deben ser tenidas en cuenta a la hora de implementar un modelo de gestión del conocimiento que colabore con la aplicación de nuevos planteamientos estratégicos en la dirección y gestión de las empresas informativas, en un proceso de adaptación constante al mercado, es decir, en un proceso de aprendizaje continuo en el que el talento organizacional, la vigilancia competitiva, las relaciones con sus públicos de interés o la innovación operativa, tecnológica y estratégica se yerguen, también, como valor diferencial.

Por último, parece evidente que, hasta que el sector de los media no se estabilice (el porcentaje 
de contratados en las empresas de menos de 10 de gestión del conocimiento va a colisionar con varios empleados y de 11 a 100 se situó, en el año 2016, en óbices obstaculizadores.

el 46,2\% -APM, 2016-), la implementación un modelo

\section{REFERENCIAS}

Albarran, A. (ed.) (2005). Handbook of media management and economics. Fairfax, Virginia: Lawrence Erlbaum.

Andersen, A. (1995). The American Productivity \& Quality Center: Knowledge Management Assessment Tool. AddisonWesley Publishing. Álvarez, N., Antolin, M., y Muñoz, M. F. (2001). La gestión del conocimiento como base de la innovación tecnológica: el estudio de un caso. Revista Espacios, 3

Andreu, R., y Sieber, S. (1999). La gestión integral del conocimiento y del aprendizaje. Economía Industrial, (2), pp. 63-72.

Asociación de la Prensa de Madrid (2016). Informe anual de la profesión periodística. Disponible en: https://goo.gl/BYBvLe (última fecha de consulta: 13/03/2018).

Barceló Llauger, M. (2001). Hacia una economía del conocimiento. Madrid: ESIC.

Bell, D. (2001). El advenimiento de la sociedad post-industrial. Un intento de pronosis social. Madrid: Alianza Editorial.

Camelo Ordaz, C. (1996). El comportamiento de las empresas frente a la comercialización en sus innovaciones: algunas reflexiones teóricas. Investigaciones Europeas de Dirección y Economía de la Empresa, 2 (2), pp. 149-170.

Carrillo-Durán, M. V. y Nuño-Moral, M. V. (2010). La documentación en la evaluación y gestión de la imagen corporativa. El profesional de la información, 19 (2) pp. 123-132. http://dx.doi.org/10.3145/epi.2010.mar.02

Choo, C. (1999). La organización inteligente. El empleo de la información para dar significado, crear conocimiento y tomar decisiones. México: Oxford.

Davenport, T. H., Prusak, L. (2001). Conocimiento en acción: Cómo las organizaciones manejan lo que saben. Buenos Aires: Pearson Education.

Douglas, P. H. (2002). Information technology is out - Knowledge sharing is in. The Journal of Corporate Accounting \& Finance, 13(4), pp. 73-77.

Drucker, P.F. (1969). The Age of Discontinuity. New York: Harper \& Row.

Falconer, J. (2000). Knowledge Management at branchpoint: will be ignore the lessons of the Al discipline theway it ignored the lessons of Ludwig Wittgenstein?. International Journal Technology Management, 20 (5-8), pp. 601-632

Freeman, R.E. (1984): Strategic Management: A Stakeholder Approach. Cambridge University Press.

García Lastra, J.M. (2012). Del modelo productivo de la era analógica al de la radio multicanal. En Gallego Pérez, J.I. y García Leiva, M. T (coords.). Sintonizando el futuro: Radio y producción sonora en el siglo XXI. Madrid: Instituto RTVE.

Gauchi Risso, V. (2012). Aproximación teórica a la relación entre los términos gestión documental, gestión de información y gestión del conocimiento. Revista Española de Documentación Científica, 35 (4) pp. 531-554.

Goleman, D., Boyatzis, R., y Mckee, A. (2002). El líder resonante crea más. Barcelona: Plaza\&Janés. 
Heidenreich, M. (2003). Die Debate um die Wissensgesellschaft. En BÖSCHEN, S., Schulz-Schaeffer, I. (eds.). Wissenschaft in der Wissensgesellschaft. Opladen. Westdeutscher Verlag. Recogido por Krüger, K. (2006). El concepto de la 'Sociedad del Conocimiento'. Biblio 3W, Revista Bibliográfica de Geografía y Ciencias Sociales, XI (683). Disponible en: https://goo.gl/Ex6ri4 (última fecha de consulta: 13/03/2018).

Jericó, P. (2000). Gestión del talento: Del profesional con talento al talento organizativo. Madrid (etc): Prentice Hall.

Kerlinger, F. N. y Lee, B. H. (2001). Investigación del comportamiento. Métodos de Investigación en Ciencias Sociales. México: Mc Graw Hill.

Krüger, K. (2006). El concepto de ‘sociedad del conocimiento'. Revista Bibliográfica De Geografía y Ciencias Sociales (Serie documental de Geo Crítica), v.XI, nº 693.

López Triana, I y Sotillo, S. (2009). La gestión de los recursos intangibles empresariales. Cuadernos de Gestión del Conocimiento Empresarial, 11. Disponible en: https://goo.gl/BWxoer (última fecha de consulta: 13/03/2018).

Mousavizadeh, M., Harden, G., Ryan, S., y Windsor, J. (2015). Knowledge Management And The Creation Of Business Value. The Journal of Computer Information Systems, 55(4), pp. 35-45.

Nonaka, I., y Takeuchi, H. (1999). La organización creadora de conocimiento: Cómo las compañías japonesas crean la dinámica de la innovación. México D.F: Oxford University Press.

Núñez Paula, I. (2004). La gestión de la información, el conocimiento, la inteligencia y el aprendizaje organizacional desde una perspectiva socio-psicológica. Acimed: revista cubana de los profesionales de la información y la comunicación en salud, 12 (3). Disponible en: https://goo.gl/iDcEDA (última fecha de consulta: 13/03/2018).

Pérez Serrano, M.J. (2015). Ingresos y gastos en la Empresa Informativa. En Núñez Fernández, V. (coord.), Peinado Miguel, F., Pérez Serrano, M.J. La Empresa Informativa en la Era Digital. Madrid: CEF, pp.123-150.

Pérez-Montoro, M. (2006). Gestión del conocimiento, gestión documental y gestión de contenidos. En Tramullas, J. (coord.). Tendencias en documentación digital. Gijón: Trea.

Picard, R. G. (2002). The economics and financing of media companies. New York: Fordham University Press.

Pringle, P. y Starr, M. F. (2004). Electronic media Management. Amsterdam: Focal Press.

Rodríguez Pallares, M. (2014). Análisis de los actuales modelos de gestión de contenidos y conocimiento en las grandes cadenas de radiodifusión españolas: SER, onda cero, COPE y RNE. Tesis doctoral: Universidad Complutense de Madrid.

Rodríguez Pallares, M. (2016). Propuesta conceptual de un modelo de gestión de contenidos y del conocmiento en la empresa radiofónica. Revista Española de Documentación Centífica, 39 (2).

Rodríguez Pallares, M. y Pérez Serrano, M.J. (2017). Innovación y transparencia: maridaje teórico, divergencia práctica. Propuesta sobre las empresas innovadoras del sector de la comunicación en España. En González-Alba, J.A. (coord.): Tendencias e innovación en la empresa periodística. Sevilla: Egregius, pp. 103-123.

Rodríguez, D. (2006). Modelos para la creación y gestión del conocimiento: una aproximación teórica. Revista Educar, 37. pp. 25-39.

Rusly, F. H., Corner, J. L., y Sun, P. (2012). Positioning change readiness in knowledge management research. Journal of Knowledge Management, 16 (2), pp. 329-355. 
Sánchez-Tabernero, A. (2000). Dirección estratégica de empresas de comunicación. Madrid: Cátedra.

Sancho, R. (2007). Innovación industrial. Revista Española de Documentación Científica, 30 (4), pp. 553-564.

Senge, P. M. (2005). La quinta disciplina: Cómo impulsar el aprendizaje en la organización inteligente. Buenos Aires. Granica.

Spender J.C., y Grant R. M. (1996). Knowledge and the firm: Overview. Strategic Management Journal (1986-1998), 17, número especial invierno, (5).

Sveiby, K. E. (1997). The New Organizational Wealth: Managing y Measuring Knowledge-based Assets. San Francisco: Berrett-Koehler Publishers, p. 9.

Sylvie, G. et al. (2008). Media Management: a casebook approach. New York: Lawrence Erlbaum Associates.

Tejedor, B. y Aguirre, A. (1998). Proyecto Logos: Investigación relativa a la capacidad de las empresas españolas. Boletín de estudios económicos, 53(164) pp. 231-249.

Todaro, M.P. (1997). Economic Development. Londres y New York: Longman.

Wikstrom, S. y Normann, R. (1994). Knowledge and value: A new perspective on corporate transformation. England: Routledge. 